\title{
Entwicklung und Einsatz von Code- generierungssoftware zur Effizienzsteigerung bei der Prozessanlagenautomatisierung
}

\author{
Johannes Silas Detje ${ }^{1}$, Jan-Eric Meißner ${ }^{2}$, Alexander Stolpmann ${ }^{3}$
}

\section{Zusammenfassung}

Die Programmierung von SPS-Systemen erfolgt hauptsächlich händisch, obwohl große Teile des Programmcodes sehr ähnlich sind und sich häufig nur durch die eingesetzten Variablen unterscheiden. Der Beitrag befasst sich mit der Entwicklung und Testung einer Codegenerierungssoftware für das TIA Portal, um den Zeitaufwand der Programmierung zu reduzieren und Flüchtigkeitsfehler zu vermeiden.

\section{Stichwörter}

SPS, Codegenerierung, TIA Portal, Anlagenautomatisierung, Softwareentwicklung

\section{Einleitung}

Fertigungs- und Prozessanlagen sind in der heutigen Zeit größtenteils vollständig automatisiert. Jedoch ist der Weg zur Automatisierung erfahrungsgemäß bisher nicht oder kaum automatisiert. Die Programmierung der Steuerungen erfolgt fast ausschließlich von Hand, obwohl sich viele Programmteile oft mit nur kleinen Variationen wiederholen. Im Rahmen einer Masterarbeit wird eine Codegenerierungssoftware zur Verwendung mit dem TIA Portal entwickelt und getestet. Das von Siemens entwickelte TIA Portal unterstützt grafisches Programmieren mit Bausteinen [1] und erlaubt einen Zugriff auf die Objekte durch die TIA Openness Schnittstelle [2]. Realisiert wird die Codegenerierungssoftware bei Integrated Lab Solutions $\mathrm{GmbH}$, einem Unternehmen, welches sich auf den Bau und die Testung von verfahrenstechnischen Anlagen im Labormaßstab spezialisiert hat [3]. Die Anlagen müssen individuell programmiert werden (STEP 7/ KOP). Jedoch werden meist Programmteile aus schon vorhandenen Projekten oder standardisierte Bausteine, für sich wiederholende Komponenten, verwendet. Ein großer Teil der Programmierarbeit besteht aus dem Kopieren von Datenbereichen und Bausteinnetzwerken und anschließendem Ersetzen der Variablenbezeichnungen. Dieses Vorgehen ist zeitintensiv und birgt Fehlerpotenzial durch falsche Eingaben. Die Anlagen besitzen eine Sicherheitslogik, welche Alarmmatrix genannt wird. Diese wird durch Strategien definiert, welche Ursachen (Auslöser) besitzen und Effekte (Interlock eines Gerätes) verursachen. Diese Strategien müssen ebenfalls zeitintensiv von Hand programmiert werden, wodurch auch hier die Gefahr einer Fehleingabe besteht. Da sie strukturiert aufgebaut sind, ist auch dort eine Automatisierung möglich und dementsprechend hilfreich. Die Codegenerierungssoftware trägt den Namen TiaHaxxor. Eine Zusammensetzung aus TIA Portal und Haxxor einem Internet-Slang für Hacker.

\footnotetext{
1 Technische Hochschule Wildau, Deutschland; Integrated Labs Solutions GmbH, Berlin

2 Integrated Labs Solutions GmbH, Berlin

3 Technische Hochschule Wildau, Deutschland
} 


\section{Stand des Wissens}

Der Ansatz einer automatisierten modularen Programmierung ist nicht neu und wurde in unterschiedlichem Umfang schon mehrfach beschrieben und/oder umgesetzt. In der Veröffentlichung von Armentia, Estevez, Orive und Marcos [4] wird eine Anlage in mechatronische Komponenten unterteilt. Eine Komponente wird in Hardware und der zugehörigen Software zerlegt. Die Modularisierung wird mithilfe von beschreibenden XML-Dateien realisiert. Dabei wird AutomationML (AML) eingesetzt, welches ein immer populärer werdender Standard ist und auch schon von Siemens und anderen Firmen verwendet und unterstützt wird. Die beschriebene Software nutzt die TIA Openness Schnittstelle. Das Hauptaugenmerk liegt jedoch in der Automatisierung der Hardware-Konfiguration. Die Veröffentlichung von Estévez, Marcos und Orive [5] beschäftigt sich mit der automatischen Generierung von Programm-Code nach dem IEC 61131-3 Software Standard. Die Arbeit von Gillert [6] beschreibt die Entwicklung eines SCL-Codegenerators für das TIA Portal. Dafür werden Vorlagen mit Platzhaltern verwendet und diese automatisch durch Werte aus einer Excel Tabelle ersetzt. Die Firmen HS Automation Software [7] und Grollmus [8] bieten kommerzielle Software an, welche die TIA Openness Schnittstelle nutzen und nach eigenen Aussagen große Teile des Engineerings automatisieren. Außerdem gibt es verschiedene Firmen, die ein eigenes Programm zur unterstützten Automatisierung nutzen, wie es bei Roske und Zanoni [9] der Fall ist. In einer Veröffentlichung aus dem Jahr 2020, die verschiedene veröffentlichte Ansätze zur Codegenerierung vergleicht, wird festgestellt, dass bisher hinreichende Beweise für Vorteile wie bessere Codequalität oder Zeitersparnis fehlen. Allerdings wurden die Methoden meist nur an einem exemplarischen Beispiel von geringem Umfang getestet [10].

\section{Prozessautomation mit TIA Portal}

Das Totally Integrated Automation (TIA) Portal ist ein Programm von Siemens, welches verschiedene Funktionen zur Automatisierung miteinander kombiniert. Es werden die grafisch orientierten Sprachen wie Kontaktplan (KOP) und Funktionsplan (FUP), aber auch textbasierte Sprachen wie Anweisungsliste (AWL) und Structured Control Language (SCL) unterstützt. Programmcode wird durch Bausteine repräsentiert, welche jeweils ein Unterprogramm darstellen. Bausteine können immer wieder an unterschiedlichen Stellen des Programms, auch verschachtelt, eingesetzt werden [1, S. 35-37]. Bild 1 zeigt, wie das Hauptprogramm nacheinander Unterprogramme (Bausteine) aufruft und abarbeitet.

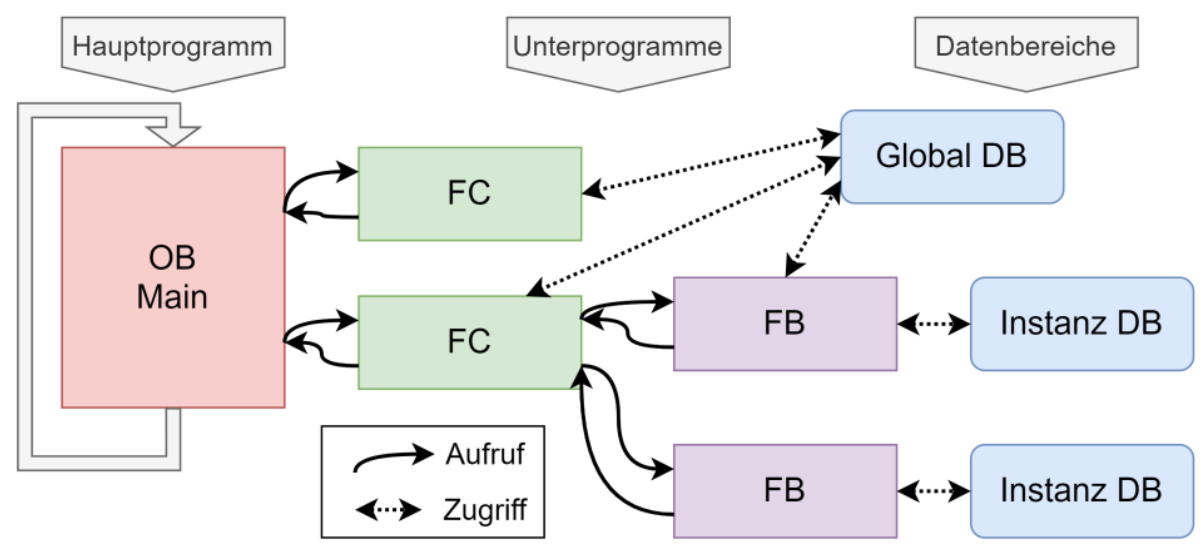

Bild 1: TIA Portal Bausteinaufrufe und Datenbereichszugriffe [Eigene Darstellung]

Organisationsbausteine (OB) werden vom Betriebssystem aufgerufen und bestimmen den Ablauf des Programms. Funktionen (FC) sind Bausteine ohne eigenen Speicher. Bei Aufruf werden Parameter von dem aufrufenden Baustein übergeben und anschließend in Form von Rückgabewerten zurückgegeben. Innerhalb von FCs kann auf globale Datenbausteine zugegriffen werden. Funktionsbausteine (FB) 
sind wie Funktionen, nur dass sie einen zugehörigen Instanz-DB besitzen und daher Daten zwischen Aufrufen speichern können. Globale Datenbausteine (DB) sind Bausteine, die nur Variablen speichern. Auf die gespeicherten Daten kann von jedem anderen Baustein durch eine absolute oder eine symbolische Adresse zugegriffen werden [1, S. 39-42].

Zusätzlich gibt es sogenannte Technologieobjekte, wie PID-Regler, Motorsteuerungen oder Zählerfunktionen. Diese werden separat konfiguriert, aber wie normale Bausteine eingefügt. Der Speicherbereich des Technologieobjektes entspricht prinzipiell einem Instanz-DB [11].

\subsection{TIA Portal Openness (API)}

TIA Portal Openness ist im Lieferumfang von STEP 7 im TIA Portal enthalten [2, S. 4]. Openness ist eine API-Schnittstelle, über die auf das TIA Portal zugegriffen werden kann und eine Möglichkeit bietet das Engineering zu automatisieren. Die Openness API ermöglicht den Zugriff auf Portaldaten, Projektdaten, PLC-Daten und HMI-Daten. Die Openness API steht über zwei DLLs zur Verfügung. Diese basieren auf dem .NET Framework 4.6.1 [2, S. 7]. Da die API auf dem .NET Framework basiert, kann eine eigene Applikation mit einer beliebigen .NET Sprache, wie C\# oder Visual Basic .NET, programmiert werden. Über die Schnittstelle kann nicht direkt in einer der Programmiersprachen (AWL, KOP, FUP, SCL) programmiert werden. Jedoch können fast alle Objekte im XML-Format exportiert und importiert werden. Für den Export eines Bausteins, muss er konsistent übersetzt sein. Für den Import ist eine Konsistenz nicht nötig. Existiert ein Baustein mit demselben Namen, wird er entweder nicht importiert oder beim Import überschrieben [12].

Das XML-Format der Bausteine entspricht einem proprietären Format von Siemens. Für eine Bearbeitung muss es entsprechend interpretiert werden.

\subsection{Projektbearbeitung bei ILS}

Automatisierungsprojekte werden bei Integrated Lab Solutions (ILS) in verschiedenen Phasen bearbeitet. Die wichtigste Phase ist das Pre-Programming. In diesem wird das SPS-Programm geschrieben und die Bedienoberfläche gestaltet. Zuvor wird eine IO-Liste erstellt.

Die IO-Liste ist bei ILS das wichtigste Mittel zur Planung und zur Kommunikation mit dem E-Zulieferer, welcher den Schaltschrank baut. Die Liste ist eine Excel-Tabelle in der alle Geräte/Komponenten der Anlage gelistet sind. Jeder Eintrag besteht aus Tagbezeichnung, Beschreibung und weiteren Angaben. Zusätzlich zur IO-Liste gibt es die Alarmmatrix, die Sicherheitslogik kommuniziert. Die bei ILS verwendete Alarmmatrix besteht aus Tabellen (Strategien), welche Ursachen und Wirkungen verknüpfen. Sie muss, wie vorgegeben, einprogrammiert werden. Jede Strategie besitzt ein Alarmlevel. Es gibt vier Alarmlevel mit unterschiedlicher Wichtigkeit. Eine Ursache wäre beispielsweise eine zu hohe Temperatur an einem Messelement. Die entsprechende Wirkung der Strategie könnte ein Abschalten des Heizelements sein.

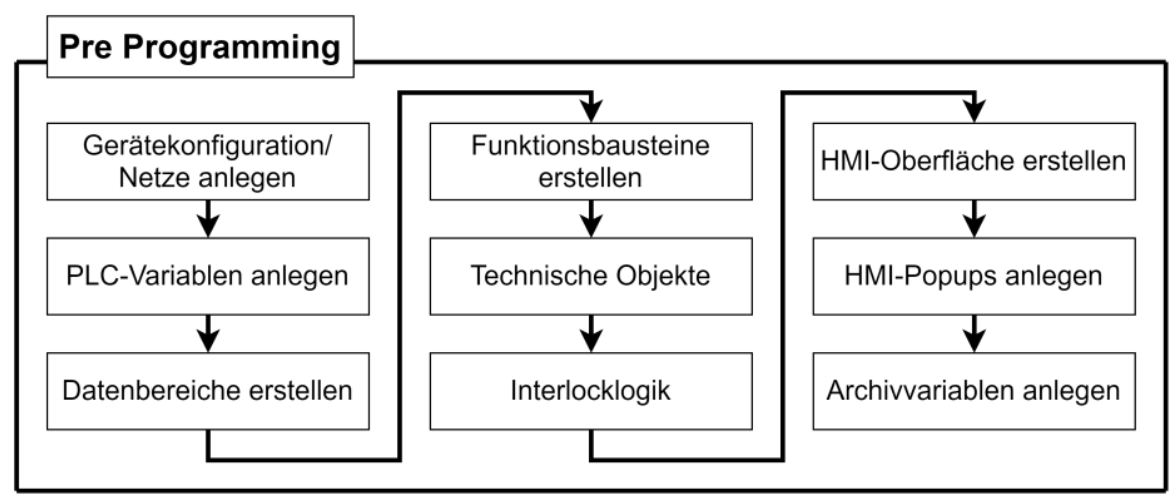

Bild 2: Bestandteile des Pre-Programming [Eigene Darstellung] 
In Bild 2 sind die Bestandteile des Pre-Programmings gezeigt. Beim Beginn der Programmierung wird zunächst die Gerätekonfiguration erstellt. Dazu gehört der Aufbau der Geräte und ihrer Baugruppen. Bei ILS wird dazu das TIA Selection Tool verwendet. Der davon generierte Export wird in das TIA Portal geladen. Es werden weitere Einstellungen vorgenommen und ggf. Profibus- oder Profinet-Geräte hinzugefügt. Anschließend werden die PLC-Variablen angelegt. Danach werden die Datenbereiche der verschiedenen Module und die Netzwerke der Module in OBs oder FBs erstellt. In diesen Netzwerken werden die Daten verarbeitet, bereitgestellt und die Steuerung des Moduls programmiert. Dafür werden meist erprobte eigene Bausteine verwendet. Sofern benötigt, werden technische Objekte erstellt und konfiguriert. Es ist bei ILS etabliert die Programmiersprache KOP zu verwenden. In Ausnahmefällen werden SCL-Netzwerke eingesetzt. Anschließend wird die HMI-Oberfläche programmiert. Der TiaHaxxor zielt darauf ab, die Schritte PLC-Variablen anlegen (und adressieren), Datenbereiche, Funktionsbausteine und Technische Objekte erstellen, zu teilautomatisieren.

\section{Softwareentwicklung}

Nachfolgend wird der Aufbau und die allgemeine Funktionsweise des Programms erklärt. Die tatsächliche Implementierung muss daraus abgeleitet werden.

\subsection{Programmaufbau}

Der TiaHaxxor besitzt eine Hauptklasse, welche den Ablauf und die Funktionen koordiniert. Die Bedienoberfläche kommuniziert mit dieser Hauptklasse. Die Kommunikation mit der TIA Openness Schnittstelle wird durch die Klasse TIAProvider realisiert. Der Bediener legt Vorlagen an, die in der Vorlagenbibliothek gespeichert werden. In den Projektinformationen wird das Projekt konfiguriert und gespeichert. Dazu gehören Variablentabellen, die jeweils mit einer Modulvorlage aus der Vorlagenbibliothek verbunden sind, und die Interlocklogik, welche eine übergeordnete Logik zwischen Modulen implementiert. Die Klassen sind in Bild 3 zu sehen.

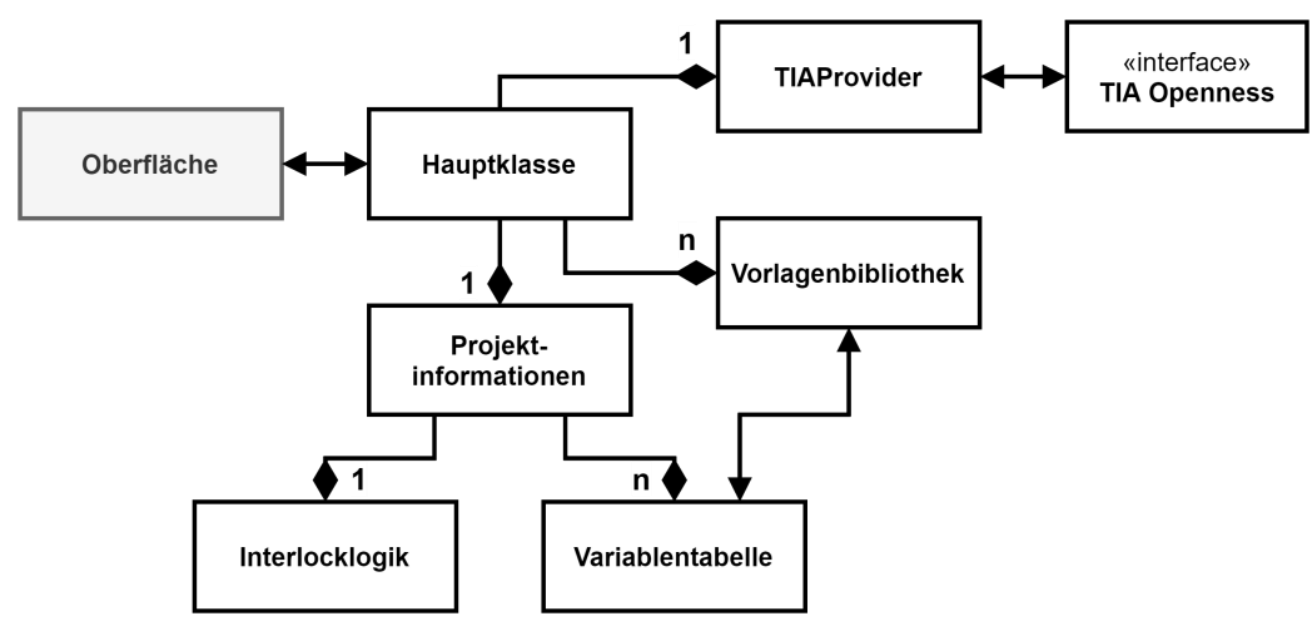

Bild 3: Allgemeiner Aufbau des Programmes [Eigene Darstellung]

\subsection{Vorlagenbibliothek}

Die Vorlagenbibliothek wird durch eine bestimmte Datenstruktur definiert (Bild 4). Das oberste Element ist die Vorlagenbibliothek. Diese besteht aus einer Liste aller Modulvorlagen und einer Liste aller möglichen Platzhalter. Eine Modulvorlage besteht wiederum aus einer Sammlung von Bausteinvorlagen. Jede Bausteinvorlage entspricht einem aus dem TIA Portal exportierten Baustein (mit wenigen 
Ausnahmen). Die Vorlage enthält einen Namen, den Name des Zielbausteins, den Dateipfad des exportierten Bausteins, den Typ der Vorlage und eine Liste alle Platzhalter, die in der Vorlage gefunden wurden.

Zur Erstellung einer Modulvorlage legt der Nutzer im TIA Portal Bausteine mit dem gewünschten Code an. An den Stellen, die dynamisch geändert werden sollen, trägt er Platzhaltervariablen ein. Dies vollzieht er für alle Bausteine, die zu einem Modul gehören (DB, FB, etc.). Anschließend legt er in der Vorlagenbibliothek eine neue Modulvorlage an und wählt mithilfe eines Dialoges die vorbereiteten Bausteine aus. Diese werden nachfolgend exportiert und in der Vorlagenbibliothek gespeichert. In manchen Fällen gibt der Nutzer noch zusätzliche Information für die Bausteinvorlage an, wie z.B. den Namen des Bausteins, der später generiert wird.

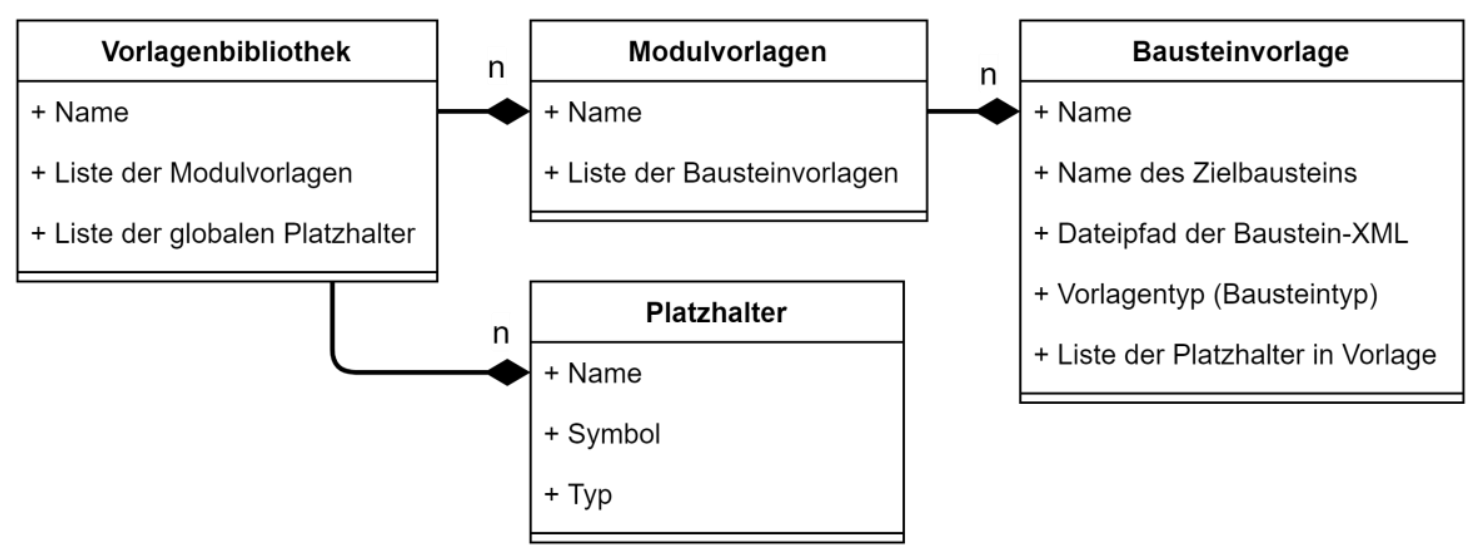

Bild 4: Struktur der Vorlagenbibliothek [Eigene Darstellung]

\subsection{Platzhalter-Typen}

Im Kompilierungsprozess werden Platzhalter durch die entsprechenden Werte ersetzt. Es gibt verschiedene Typen von Platzhaltern. In Tabelle 1 sind die unterschiedlichen Typen gelistet. Grundsätzlich würde der TiaHaxxor keine Platzhalter-Typen benötigen. Die Typen dienen der besseren Bedienung, weil sie die Eingaben des Benutzers limitieren und so das Fehlerrisiko minimieren. Der Typ Main ist für das Haupttag. Es muss einen Platzhalter des Typen Main geben. Der Typ InnerRef ist für Platzhalter, die durch eine Referenz auf ein Haupttag ersetzt werden müssen. In diesem Fall ein Haupttag derselben Modulvorlage. Der Typ CrossRef ist ähnlich mit dem Unterschied, dass die Referenz auf ein Haupttag einer anderen Modulvorlage erfolgt.

Tabelle 1: Platzhalter

\begin{tabular}{|l|l|}
\hline Platzhalter Typen & Beschreibung \\
\hline Default & Ohne Einschränkungen. Wenn es gefunden wird, dann wird es durch Wert ersetzt. \\
\hline Main & Typ für Haupttag; nur ein Haupttag gleichzeitig erlaubt \\
\hline InnerRef & Referenz auf ein Haupttag derselben Modulvorlage \\
\hline CrossRef & Referenz auf ein Haupttag einer anderen Modulvorlage \\
\hline Boolean & Wert darf nur True oder False sein \\
\hline Comment & Für Kommentare; darf nur in Beschreibungen, Titel und Kommentaren vorkommen. \\
\hline
\end{tabular}




\subsection{Interlocklogik}

Auf Grundlage der Alarmmatrix wird die Interlocklogik vom TiaHaxxor in das Steuerungsprogramm implementiert. Dabei wird die übliche Struktur der Programmierung beibehalten und nur automatisch die entscheidenden Elemente eingefügt. Dafür muss die Interlocklogik im TiaHaxxor definiert werden. Der Bediener gibt die Strategien in einer Maske ein oder er nutzt eine Erweiterung, die eine XML-Datei aus der Alarmmatrix-Excel-Tabelle erstellt. Die automatische Interlocklogik kann nur für einen Interlock verwendet werden, der mithilfe eines Bits gesetzt wird. Komplizierte Wirkungen müssen nachträglich von Hand programmiert werden. In der Vorlage wird an einer Position eine Markierung, in Form eines Kontaktes, mit der Beschriftung „[InterlockTag]“ eingefügt. An dieser Stelle werden vom Programm automatisch auslösenden Strategien eingefügt, die dann das Interlockbit des Moduls setzen. Der Unterschied zu einem Platzhalter besteht darin, dass die Variable nicht geändert wird, sondern an der Stelle weitere Elemente eingefügt werden. Ob eine Strategie ausgelöst wird, wird in einem Alarmlevelbaustein definiert. Dort werden alle Ursachen verknüpft und steuern, ob die Strategie aktiv ist. Die Markierung heißt dort „[CauseTag]“. Ursachen sind (Alarm-)Zustände von Modulen. Da die Benennung der Datenbereiche einer gewissen Struktur folgt, kann die Variablenadresse der Ursache generiert werden.

\subsection{Kompilierung}

Unter Kompilieren versteht sich in diesem Kontext, dass Zusammenfassen der Informationen aus Vorlagen, Variablentabellen und Interlocklogik und dem Erstellen der Bausteine für das TIA Portal. Die Kompilierung besteht aus mehreren Schritten. Essenziell ist dabei die Erstellung eines Kompilierungsrezeptes. Es wird zunächst erstellt und besteht aus Schritten, welche anschließend nacheinander interpretiert und abgearbeitet werden.

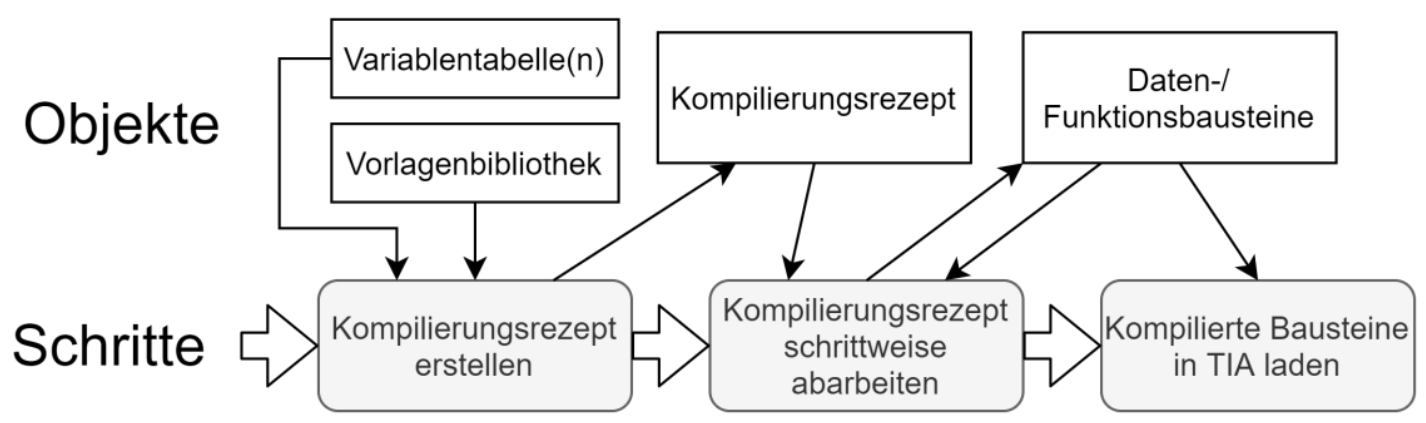

Bild 5: Ablauf Kompilierung [Eigene Darstellung]

In Bild 5 ist der Ablauf der Kompilierung schematisch dargestellt. Unten befinden sich die Schritte, oben die Objekte, mit welchen der Schritt interagiert. Als Ausgangsobjekte existieren Variablentabellen, also Tabellen in der die Modultags, Kommentare, Adressen und Kreuzreferenzen eingetragen sind und die Vorlagenbibliothek, in der sich die Vorlagen mit Platzhaltertags befinden. Aus diesen beiden Objekten wird das Kompilierungsrezept erstellt. Jeder Schritt des Rezeptes enthält dabei eine Bausteinvorlage und die Anweisung, wie sie manipuliert werden soll; also die Bezeichnung der Platzhalter und die Werte, mit welchen sie zu ersetzen sind, aber auch die Informationen, welche Elemente wo eingefügt werden sollen. Das Rezept wird vom Hauptprogramm erstellt. Im nächsten Schritt wird das Rezept vom TIAProvider interpretiert und schrittweise abgearbeitet. Dabei werden Daten- und Funktionsbausteine erstellt und bearbeitet. Wenn ein Template als Ziel einen Baustein hat, der schon durch einen anderen Schritt erstellt wurde, dann wird dieser wieder geladen und erweitert. Sind alle Schritte des Kompilierungsrezeptes abgearbeitet, dann werden alle erstellten Bausteine in das TIA Portal geladen. 


\subsection{Benutzeroberfläche}

Bei dem Design der Benutzeroberfläche ist es wichtig die Erwartungshaltung des Nutzers zu beachten. Dieser kennt schon zahlreiche andere Programme und erwartet einen ähnlichen Aufbau und ordnet die Funktionalität entsprechend ein. Gerade bei Einsatz einer Menüleiste ist die in Anwendungen geläufige Strukturierung zu beachten.

Prinzipiell besteht die Anwendung aus einem Hauptfenster und verschiedenen Nebenfenstern zum Konfigurieren der Modulvorlagen und der Interlocklogik. Dazu kommen verschiedene kleinere Dialoge. In Bild 6 ist das Hauptfenster zu sehen. Dieses öffnet sich, wenn der TiaHaxxor ausgeführt wird. Das Schließen des Fensters beendet das Programm.

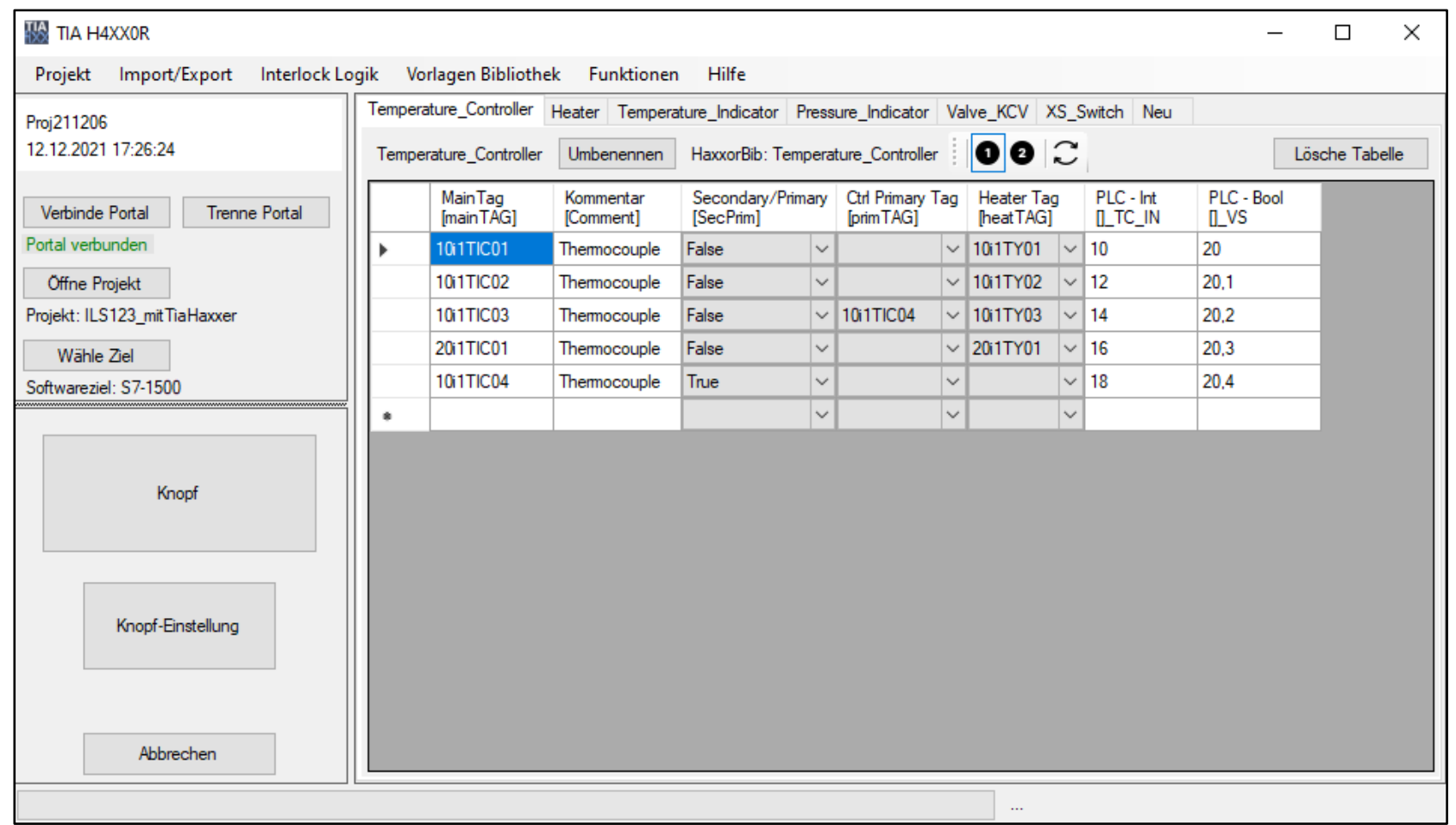

Bild 6: Hauptfenster des TiaHaxxors [Eigene Darstellung]

Das Fenster besitzt oben eine Menüleiste, über die verschiedene Funktionen abgerufen werden können. Im linken oberen Bereich befinden sich Schaltflächen, um die Anwendung mit dem TIA Portal zu verbinden und wieder zu trennen. Darunter befinden sich Statusanzeigen und Meldungen zur aktuellen Verbindung und dem aktuellen Softwareziel. Im linken unteren Bereich befinden sich drei Schaltflächen. Die große Schaltfläche namens „Knopf“ startet die Kompilierung und das Einfügen des Codes ins Portal. Die Benennung der Schaltfläche wird gewählt, weil lustige Bezeichnungen sehr einprägsam sein können und es eine Anspielung auf das Klischee ist, dass Programmierer bzw. Automatisierer für die Programmierung nur auf „ein paar Knöpfe drücken“ müssen. Über die Schaltfläche „Knopf-Einstellungen“ wird konfiguriert, welche Tabellen kompiliert und geladen werden sollen. Darunter befindet sich eine Schaltfläche zum Abbrechen des Kompilierungsvorgangs. Unten im Fenster befindet sich eine Fortschrittsleiste, die den aktuellen Fortschritt bei der Kompilierung anzeigt.

Im rechten Teil des Fensters befindet sich eine Tab-Leiste, mit der unterschiedliche Variablentabellen aufgerufen werden können. In der Variablentabelle werden alle Variablen (Tagbezeichnungen) eines Moduls eingetragen, welche später die Platzhalter ersetzen werden.

Das Fenster der Vorlagenbibliothek wird über den entsprechenden Menüeintrag aufgerufen. In Bild 7 ist das Fenster dargestellt. Das Fenster besteht aus drei Bereichen. Im rechten Bereich befindet sich die „Blank Tag Masterliste“, also die Liste aller verwendbaren Platzhalter mit ihren Namen und Typen. 
Diese kann durch drei Schaltflächen „Neu“, „Bearbeite“ und „Lösche“ bearbeitet und erweitert werden. Zu Bearbeitung wird ein kleines Dialogfenster eingeblendet. Dort werden das Symbol (die Zeichenfolge des Platzhalters) und der Name des Blanktags angeben. Für den Typ steht eine Kombobox zu Verfügung. Der Typ limitiert die später zulässigen Werte in der Variablentabelle und spezifiziert ihre Verarbeitung.

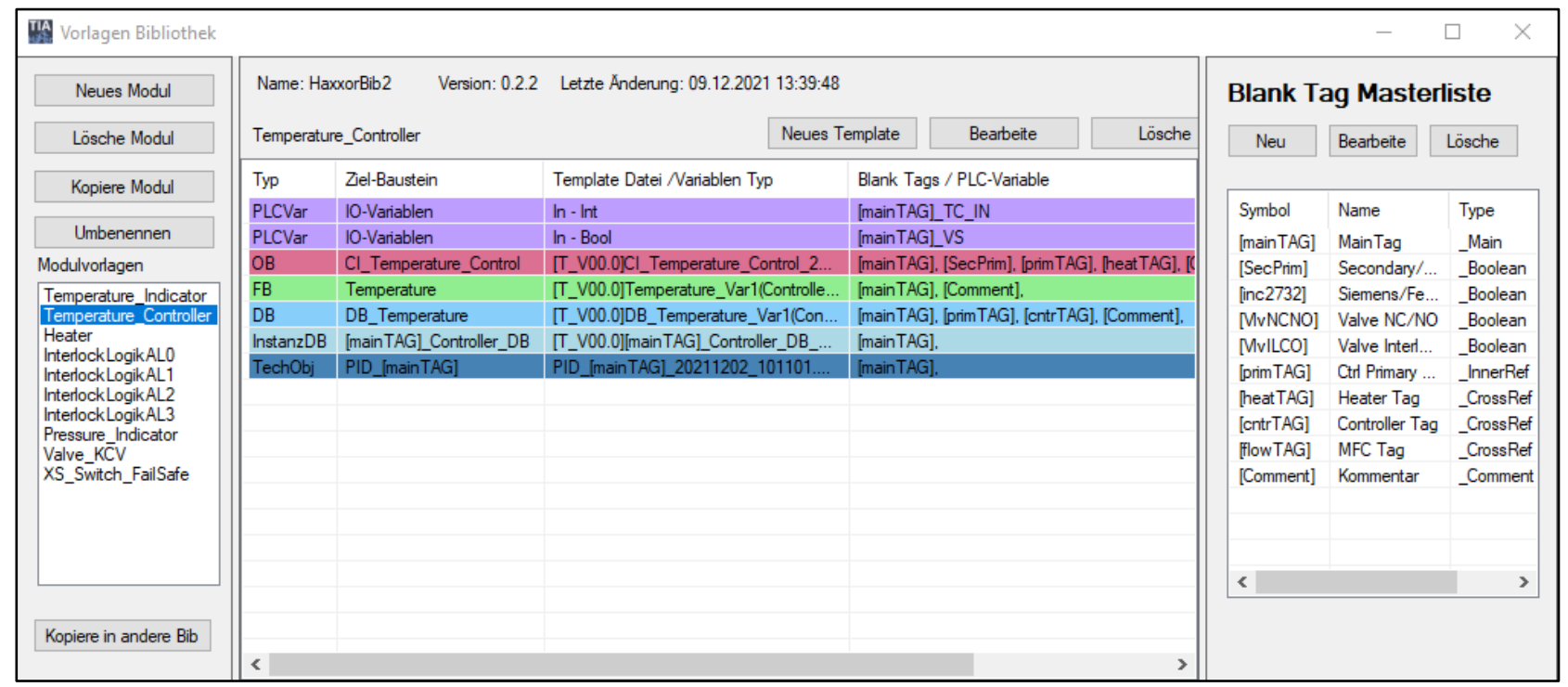

Bild 7: Fenster Vorlagenbibliothek [Eigene Darstellung]

Im linken Bereich befindet sich eine Liste mit allen Modulvorlagen. Darüber befinden sich wieder Schaltflächen zur Bearbeitung. Wird in der Liste eine Modulvorlage ausgewählt, wird diese im mittleren Bereich geöffnet und die zugehörigen Bausteinvorlagen angezeigt. Oben im mittleren Bereich stehen der Name der Vorlagenbibliothek, die Versionsnummer und das Datum der letzten Änderung. Mit den Schaltflächen „Neues Template“, „Bearbeite“ und „Lösche“ können Bausteinvorlagen erstellt und verwaltet werden. In der unteren Tabelle werden sie angezeigt.

Wird eine neue Bausteinvorlage erstellt, dann ist eine Verbindung zum TIA Portal zwingend notwendig. Es öffnet sich ein Dialogfenster in welchem ein TIA Portal-Objekt ausgewählt werden kann. Es stehen alle Bausteine, Geräte und PLC-Variablen zur Auswahl. Das dort ausgewählte Objekt dient als neue Vorlage und wird aus dem TIA Portal exportiert. Anschließend öffnet sich ein Dialogfenster der Vorlage. In ihm wird der Typ der Vorlage und gefundene Platzhalter angezeigt. Es kann ein vom ursprünglichen Namen abweichender Name für den Zielbaustein eingegeben werden.

\section{Einsatz und Test}

Um den Nutzen des TiaHaxxors zu bewerten, wird die Zeitersparnis an einem pseudorealen Projekt, genannt ILS123, gemessen. Dafür wird das Projekt einmal ohne und einmal mit TiaHaxxor teilweise umgesetzt. Das Projekt ist einem echten Projekt entlehnt, wurde aber hinsichtlich des Umfangs und der Funktionalität verändert.

\subsection{Testszenario}

In dem Testszenario sind keine Neuentwicklungen nötig und alle Komponenten sind bekannt und standardisiert. Die Programmierung einer Benutzeroberfläche (HMI) wird völlig vernachlässigt. Das Projekt ILS123 besteht aus: 24 Massendurchflussregler; 4 ESD-Schalter; 9 Türschalter; 11 Heizkreise 
bestehend aus Regler, Heizung und 2 Thermoelementen; 29 Ventile; 11 Sicherungskontakte; 10 Temperaturmessern; 3 Druckmessern und diversen anderen Komponenten. Die Komponenten sind in einer IO-Liste festgehalten. In dieser stehen Tagbezeichnung und verwendete Eingänge bzw. Schnittstelle. Die Adressen der PLC-Variablen sind vorab definiert. Zusätzlich wird die Alarmmatrix implementiert. Auch diese wurde dem realen Projekt entlehnt, stellenweise aber verändert. Sie besteht aus 16 Strategien mit unterschiedlichem Umfang (durchschnittlich 4 Ursachen und 2 Wirkungen). Die Effekte bestehen aus den Standard-Interlockeffekten. Es gibt keine besonderen Strategien, die eine erweiterte Programmierarbeit erfordern. Für die händische Programmierung wird das bei ILS übliche Vorgehen genutzt. Bei der Programmierung mit dem TiaHaxxor werden alle zur Verfügung stehenden Möglichkeiten genutzt. Die benötigten Modulvorlagen sind vorab konfiguriert und einsatzbereit. Eine Abschätzung der Projektprogrammierung ergibt, dass der Test ungefähr 45 Prozent der vollständigen Projektprogrammierung umfasst.

\subsection{Auswertung}

In Tabelle 2 sind die Zeiten der Programmierung aufgetragen. Es wurde zwischen der normalen Programmierung und der Implementierung der Interlocklogik unterschieden. Von Hand dauert die gesamte Programmierung 8 Stunden, mit dem TiaHaxxor 2,5 Stunden. Das ergibt eine Zeitersparnis von 5,5 Stunden.

Tabelle 2: Zeiten Testprojekt

\begin{tabular}{|l|l|l|l|}
\hline & Programmieren von Hand & Programmieren mit TiaHaxxor & Differenz \\
\hline Bausteine & $07 \mathrm{~h} 1 \mathrm{~min}$ & $2 \mathrm{~h} 11 \mathrm{~min}$ & $4 \mathrm{~h} 50 \mathrm{~min}$ \\
\hline Interlocklogik & $01 \mathrm{~h} 3 \mathrm{~min}$ & $20 \mathrm{~min}$ & $43 \mathrm{~min}$ \\
\hline Gesamt & $8 \mathrm{~h} 4 \mathrm{~min}$ & $2 \mathrm{~h} 31 \mathrm{~min}$ & $5 \mathrm{~h} 33 \mathrm{~min}$ \\
\hline
\end{tabular}

Die Programmierung von Hand ist ermüdend und repetitiv. Es treten immer wieder Flüchtigkeitsfehler auf, sodass manche Bausteine ein zweites Mal überarbeitet werden müssen. Auch fällt die Kommentierung der Netzwerke teilweise recht spärlich aus. Die Programmierung mit dem TiaHaxxor ist weniger wiederholend. Variableninformation müssen nur einmal angegeben werden. So minimieren sich Flüchtigkeitsfehler. Treten dennoch welche auf, sind sie leichter zu finden und zu beheben. Die Implementierung der Interlocklogik ist mit dem TiaHaxxor schneller und unkompliziert. Durch die automatische konsequente Benennung von Netzwerken und Kommentaren wird eine spätere händische Anpassung und Erweiterung der Programmierung erleichtert. Jedoch erweist sich die Bedienung des TiaHaxxors stellenweise als kompliziert. Es erfordert mehrere Anläufe bis das Projekt, in welches der Code eingefügt werden muss, dahingehend angepasst ist, dass es keine Konflikte mit den Modulvorlagen hinsichtlich der Projektsprachen und Datentypen bzw. Standardbausteinen gibt.

\section{Fazit}

Die Entwicklung des TiaHaxxors kann als erfolgreich gewertet werden. Die Verwendung einer Vorlagenbibliothek erweist sich als sinnvoll und praktisch. So ist die Codegenerierung nicht auf eine konkrete Anwendung beschränkt, sondern kann, im Rahmen der beschriebenen Möglichkeiten, beliebig erweitert werden. Einzig die Art der Interlocklogik ist beschränkt auf das beschriebene Konzept, könnte aber in Zukunft erweitert werden. Durch die erfolgte Entwicklung von Funktionen zur unkomplizierten Manipulation von KOP-Netzwerken ist eine noch komplexere Codegenerierung in Zukunft denkbar. Die Entwicklung ist prinzipiell abgeschlossen, kann aber fortgesetzt werden. Die Oberfläche 
besitzt noch Verbesserungspotenzial und sollte hinsichtlich Bedienbarkeit und Benutzerführung verbessert werden. Durch die Verwendung von Platzhaltertypen, können Verknüpfungen zwischen Modulen erstellt und hinsichtlich ihrer Gültigkeit geprüft werden. So können beispielsweise Kaskadenregler konfiguriert werden. Der Test am Beispielprojekt zeigt, dass eine Zeitersparnis messbar und nennenswert ist. Auch die verbesserte Codequalität ohne Flüchtigkeitsfehler hat einen großen Nutzen und trägt zur Effizienzsteigerung bei. Der TiaHaxxor wird in Zukunft bei ILS eingesetzt werden. Das Anlegen und Pflegen der Vorlagenbibliothek könnte sich als Zeitintensiv erweisen und es sollte ermittelt werden, ab welcher Projektgröße oder Projektanzahl sich die Entwicklung und das Betreiben eine Codegenerierungssoftware wirklich lohnt.

\section{Literatur}

[1] Siemens, SIMATIC STEP 7 und WinCC Engineering V16 Systemhandbuch: PLC programmieren. [Online]. Verfügbar unter: https://support.industry.siemens.com/cs/attachments/109773506/STEP_7_WinCC_V16_deDE_de-DE.pdf (Zugriff am: 20. April 2021).

[2] Siemens, TIA Portal Openness: Einführung und Demoanwendung. [Online]. Verfügbar unter: https://support.industry.siemens.com/cs/document/108716692/tia-portal-openness\%3Aeinf\%C3\%BChrung-und-demoanwendung (Zugriff am: 26. April 2021).

[3] Integrated Lab Solutions, Website | Integrated Lab Solutions (Zugriff am: 21. September 2021).

[4] A. Armentia, E. Estevez, D. Orive und M. Marcos, „A Tool Suite for Automatic Generation of Modular Machine Automation Projects“ in 2018 IEEE 16th International Conference on Industrial Informatics (INDIN), Porto, 72018, S. 553-558, doi: 10.1109/INDIN.2018.8472085.

[5] E. Estévez, M. Marcos und D. Orive, „Automatic generation of PLC automation projects from component-based models“ (En;en), Int J Adv Manuf Technol, Jg. 35, 5-6, S. 527-540, 2007, doi: 10.1007/s00170-007-1127-4.

[6] A. E. Gillert, „Entwicklung eines SCL-Codegenerators für die Entwicklungsumgebung TIA Portal“. Bachelorarbeit, 2016. [Online]. Verfügbar unter: http://www.lab4inf.fh-muenster.de/lab4inf/docs/thesis/BA_Gillert_20160708.pdf

[7] HS Automation Software, PLC Code Generator: Code Generator für das TIA Portal. [Online]. Verfügbar unter: https://www.hs-automation-software.de/plccodegenerator.php (Zugriff am: 14. April 2021).

[8] Grollmus -, Factory Automation Studio | TIA Portal - Grollmus. [Online]. Verfügbar unter: https://www.grollmus.de/factory-automation-studio/ (Zugriff am: 21. September 2021).

[9] M. Roske und P. Zanoni, Automatisierung von Engineering-Aufgaben bei der SPSProgrammierung im TIA Portal. [Online]. Verfügbar unter: https://www.all-electronics.de/automatisierung/automatisierung-von-engineering-aufgaben-bei-der-sps-programmierung-im-tiaportal.html (Zugriff am: 14. April 2021).

[10] H. Koziolek, A. Burger, M. Platenius-Mohr und R. Jetley, „A classification framework for automated control code generation in industrial automation“, Journal of Systems and Software, Jg. 166, S. 110575, 2020, doi: 10.1016/j.jss.2020.110575.

[11] Siemens, SIMATIC STEP 7 und WinCC Engineering V16 Systemhandbuch: Technologiefunktionen einsetzen. [Online]. Verfügbar unter: https://support.industry.siemens.com/cs/attachments/109773506/STEP_7_WinCC_V16_deDE_de-DE.pdf (Zugriff am: 20. April 2021).

[12] Siemens, Openness: Projekterstellung automatisieren: Online help printout. [Online]. Verfügbar unter: https://support.industry.siemens.com/dl/dl-media/802/109773802/att_1007203/v6/131611131147/de-DE/index.html (Zugriff am: 26. April 2021). 